\title{
Fas and FasL expression on cells of two transplantable melanoma lines according to their different biological properties
}

\author{
Katarzyna Zielińska, Krystyna Kozłowska, Mirosława Cichorek, \\ Małgorzata Wachulska
}

Department of Embryology, Medical University of Gdańsk, Poland

\begin{abstract}
Fas and FasL interaction induces apoptotic cell death. In immunocompetent cells it plays a crucial role in the effector functions of the cells and in the regulation of host immune response. In tumours (e.g. melanoma), FasL expression possibly counteracts the Fas-positive effector T cells that infiltrate into tumours, and consequently the Fas/FasL interaction can contribute to the escape of tumour cells from the systemic immune response. In this study we examined differences in Fas and FasL expression on cells from the hamster melanotic melanoma line (Ma) and a more aggressive amelanotic melanoma line $(\mathrm{Ab})$. We also tried to find out whether the Fas/FasL expression induces an ability to undergo spontaneous apoptosis in these two transplantable melanoma lines. Our previous studies have shown that cells of the Ma line have a higher ability to undergo spontaneous apoptosis than cells of the Ab line. Isolated transplantable melanoma cells were incubated for 4 and 24 hours and after that time the expression of Fas and FasL was estimated by flow cytometry. The results show that there was no Fas expression, although FasL was detected on both melanoma cell lines. Therefore the data reported by other authors indicate that a lack or a low level of Fas expression and an ectopic expression of FasL on melanoma cells can be an escape mechanism of the tumour, to avoid host immune responses. The content of FasL-positive melanotic melanoma cells was higher than in amelanotic melanoma cells and increased with the prolongation of the incubation time. FasL expression on amelanotic melanoma cells was detected after 24 hours at a level similar to that on melanotic melanoma cells after 4 hours incubation time. FasL expression on melanoma cells can induce apoptosis in cytotoxic T lymphocytes and NK cells which are responsible for tumour cells elimination. The results obtained suggest that the Fas/FasL system does not play any significant role in spontaneous apoptosis of two melanoma cell lines. But these results may indicate the presence of immune privilege of tumour cells with FasL expression.
\end{abstract}

Keywords: Fas - FasL - Transplantable melanoma

\section{Introduction}

Fas, also called CD95 or APO-1, which belongs to TNF Superfamily of membrane death receptors, induces cell death by binding with FasL (Fas Ligand). Fas was the first death receptor to be described as an apoptosis-inducing receptor [1]. FasL (CD95L, APO$1 \mathrm{~L}$ ) is expressed on activated T cells and NK cells, and mediates immune responses [2]. Membrane-bound FasL induces apoptotic cell death of Fas-positive cells [3]. FasL overexpression is involved in the formation of immune privilege. The immune-privileged sites such as the anterior chamber of the eye, the Sertoli

Correspondence: K. Zielińska, Department of Embryology,

Medical University of Gdańsk, Dębinki 1 St, 80-211 Gdańsk,

Poland; tel.:/fax.: (+4858) 3491495, e-mail: kazielin@amg.gda.pl cells in the testis, the brain, the ovary and the uterus and placenta during pregnancy, constitutively express high amounts of FasL and thus can protect themselves from immune reactions [4]. FasL, expressed locally and released from cells of those organs, where systemic immunity is poorly induced, immediately kills infiltrating immunocompetent cells and induces apoptosis of Fas-positive activated $\mathrm{T}$ cells that infiltrate into the tissues $[4,5]$. Fas-mediated apoptosis is an important mechanism in cell-mediated immune response [1].

As shown by previous investigations, Fas and its ligand, FasL, are involved in the elimination of activated lymphocytes by apoptosis, to down-regulate immune responses. Thereby the Fas/FasL system plays a crucial role in the maintaining of the immune homeostasis, and also in the regulation of immune response against tumours [6]. However, high FasL expression 
has been consistently observed in different types of human tumours, also in melanomas. The loss of cell surface Fas expression has been described for melanomas, breast and oesophageal tumours, leukemia, lymphomas $[7,8,9]$. On the other hand, it has been reported that a variety of malignant tumours, including: carcinomas of the colon, breast, ovary, urinary bladder, lung, head and neck, melanoma, astrocytoma, show an increased FasL expression $[7,10,11]$. In the case of melanoma, an increased FasL expression is correlated with aggressiveness and bad prognosis. Due to FasL up-regulation and Fas down-regulation, malignant tumours could evade immune control via induction of apoptosis of effector immune cells, like cytotoxic $\mathrm{T}$ cells and NK cells, in a process that has been called 'tumour counterattack' $[4,12,13]$.

Apoptosis plays an important role in the regulation of the number of cells and is controlled by a variety of intracellular and extracellular signals: via receptors (extrinsic pathway) and mitochondria (intrinsic pathway) $[14,15,16]$. Ligands bind with membrane-bound death receptors and activate caspases. Apoptotic signalling appears when the Fas/FasL system binds to the Fas-associated death domain (FADD) in the cytoplasm, with a subsequent activation of caspase 8 , and initiates the executionary phase of apoptosis. The signal may be delivered to mitochondria and is amplified due to the release of proapoptotic molecules such as cytochrome c $[1,14]$. This signal transduction pathway leads to apoptotic cell death.

Some invasive tumours, like melanoma, characterized by a Fas negative/FasL positive phenotype may be connected with a worse prognosis [17]. Those results inclined us to investigate the Fas/FasL system and its role in cells of two lines of hamster transplantable melanomas: melanotic and amelanotic, of common origin but differing in many biological features.

Thus, the aim of the present investigation was to analyse the expression of Fas and FasL on cells of two transplantable melanoma lines and possible changes which might be associated with a spontaneous alteration of the native melanotic melanoma form into amelanotic one, accompanied by tumor progression (with a higher growth rate and shorter animal survival time). Our previous observations concerning the ability of melanoma cells to undergo spontaneous apoptosis $[18,19,20]$ have shown that melanotic melanoma cells (Ma) have a higher ability to undergo spontaneous apoptosis than amelanotic melanoma cells $(\mathrm{Ab})$. In the present study we would like to find a relationship between the expression of the Fas/FasL system and the ability of melanotic and amelanotic melanoma cells to undergo spontaneous apoptosis and a possibility to use the Fas/FasL death pathway for therapeutic strategies for this highly malignant tumour.

\section{Materials and Methods}

Animals. 3-4 months old male Syrian (golden) hamsters Mesocricetus auratus Waterhouse, were purchased from the Central Animal Facilities of Silesian Medical University (Katowice, Poland). The experiments' procedures were approved by the Animal Ethics Committee of Medical University of Gdansk $(16 / 2007 ; 18.06 .2007)$ and confirmed to the National Health and Medical Research Council's guide for the care and use of laboratory animals.

Transplantable melanomas. Cells for studies were isolated from transplantable melanotic and amelanotic melanomas [21].

Melanotic melanoma line (Ma). The original transplantable melanotic melanoma line derived from a spontaneous melanoma of the skin which had appeared in a breed of golden hamsters in 1959.

Amelanotic melanoma line (Ab) originated from the melanotic form (Ma) by a spontaneous alteration and had appeared in a breed of golden hamsters in 1963. Both transplantable melanoma lines were described by Bomirski in 1977 as melanotic (Ma) and amelanotic (Ab) melanoma lines [22,23]. Loss of pigment in the $\mathrm{Ab}$ line was accompanied by changes in many biological features - faster tumour growth rate, shorter animal survival time, cell ultrastructure changes and changes in antigenicity and immunogenicity $[21,22]$. Once established, these melanomas possessed a considerable degree of phenotypic stability over decades of passaging [21]. Since their discovery, each melanoma line is maintained in vivo by consecutive, subcutaneous transplantations of tumour material every $21(\mathrm{Ma})$ or $11(\mathrm{Ab})$ days. At that time 2-3 animals were used for the experiments in each experimental group of animals.

Isolation of melanotic and amelanotic melanoma cells. Melanoma cells were isolated for each experiment from solid tumours by mechanical dissociation of tissue. Then melanoma cells were isolated by means of a mixture 9\% Ficoll 400 (Pharmacia, Sweden) and 75\% uropoline (Polpharma, Poland) in ratio 7.6:1 as described previously $[19,24]$. The suspension contained $95-98 \%$ of viable cells (estimated by trypan blue test).

Incubation of cells. Isolated melanoma cells at the concentration of $2 \times 10^{6} / \mathrm{ml}$ were incubated in RPMI 1640 (culture medium; Biomed, Poland) with $10 \%$ FCS (fetal calf serum; Gibco) and antibiotics in 6-well plates (Corning) at $37^{\circ} \mathrm{C}$, for 4 and 24 hours.

Detection of Fas and FasL expression by flow cytometry. After incubation time cells were harvested, washed with PBS and immunostained with: hamster anti-mouse Fas monoclonal antibody and mouse anti-mouse FasL monoclonal antibody conjugated with phycoerythrine (PE) $\left(1 \mu \mathrm{g} / 10^{6}\right.$ cells; BD Pharmingen, CA, USA). Cells were incubated for 40 minutes, in dark. Then, cells were washed with PBS three times and the intensity of fluorescence was measured with the FACS Calibour (Becton Dickinson, NJ, USA).

The background isotype control of non-specific binding to the cells was PE-labelled mouse $\operatorname{IgG}_{2 b}$ (BD Pharmingen, CA, USA). Hamster splenocytes for Fas and hamster peritoneal macrophages for FasL were used as a positive control. After gating out small-sized (e.g. noncellular debris) object, fluorescence obtained from 10000 - 20000 cells per sample, were analysed with WinMDI 2.6 Software (J. Trotter, The Scripps Institute, La Jolla, CA, USA) and were shown as percentage of Fas- or FasL-positive cells.

Statistical analysis. Data expressed as mean \pm SD were statistically estimated by the non-parametric Mann-Whitney U test using 

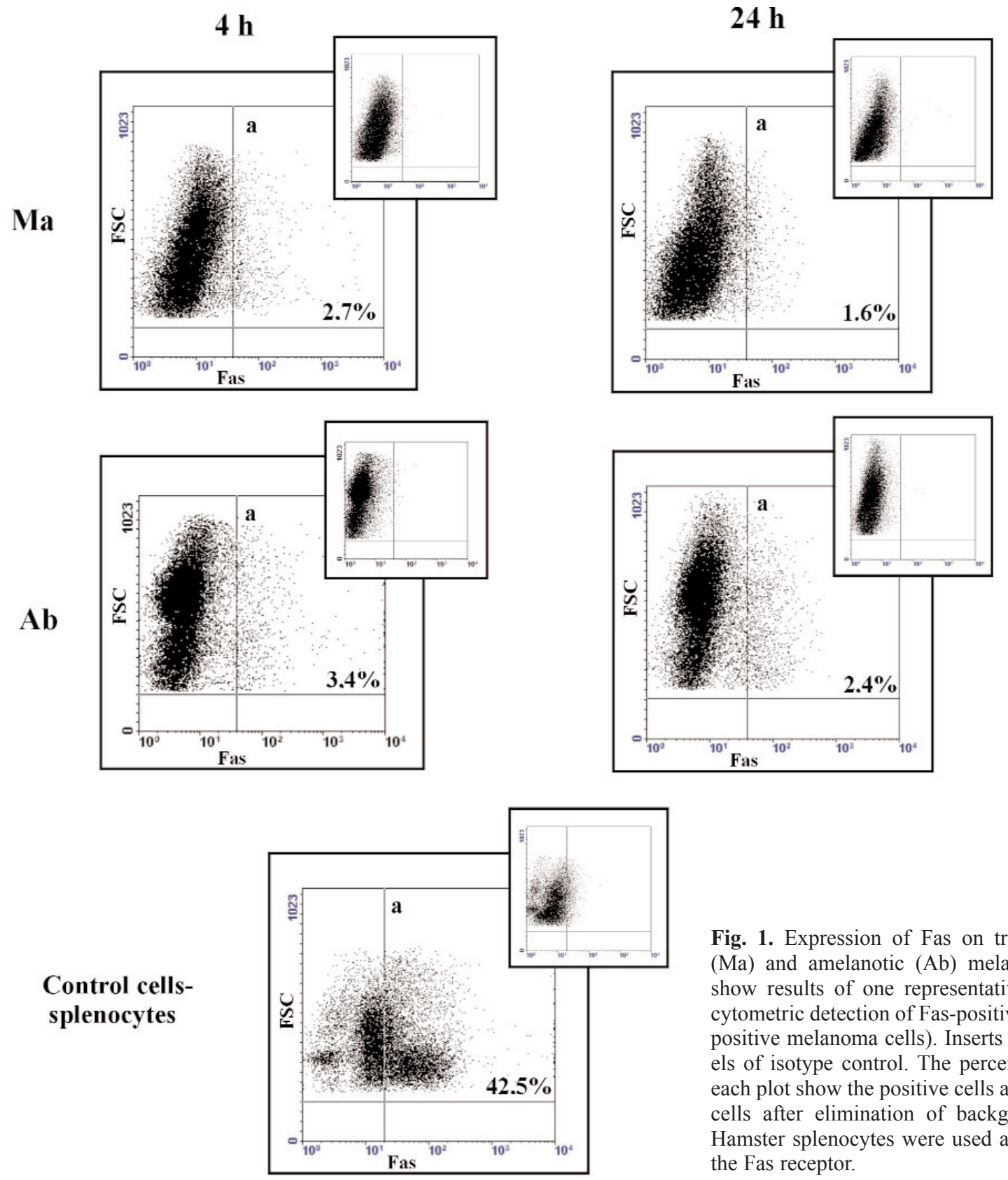

Fig. 1. Expression of Fas on transplantable melanotic (Ma) and amelanotic (Ab) melanoma cells. The plots show results of one representative experiment of flow cytometric detection of Fas-positive cells (a - area of Faspositive melanoma cells). Inserts show fluorescence levels of isotype control. The percentages in the corner of each plot show the positive cells among the population of cells after elimination of background isotype control. Hamster splenocytes were used as a positive control for the Fas receptor.

StatSoft, Inc. (2005) STATISTICA (data analysis software system), version 7.1. www.statsoft.com. The value of $\mathrm{p}<0.05$ was considered to represent a statistically significant difference.

\section{Results}

\section{Fas expression on the transplantable melanoma cell lines}

The flow cytometric results of hamster transplantable melanoma cells Fas antibody binding are shown in Fig. 1.

Both, on melanotic melanoma (Ma) cells and on amelanotic melanoma $(\mathrm{Ab})$ cells, after 4 and 24 hours
Fas expression was not observed (Table 1). Only a few melanotic and amelanotic melanoma cells (Table 1) bound anti-Fas antibody in comparison with hamster splenocytes - among which $42.5 \%$ were Fas-positive (Fig. 1).

\section{FasL expression on the transplantable melanoma cell lines}

The results of FasL antibody binding by examined melanoma cells are shown in Fig. 2.

FasL was detected on both melanoma cell lines (Table 1). In hamster peritoneal macrophages, used as 
Table 1. Percentage of Fas- and FasL-positive cells of two transplantable melanoma: melanotic (Ma) and amelanotic (Ab) lines. The values are means \pm SD of 4 - 7 experiments for Fas and FasL expression. Statistical analysis by the Mann-Whitney U test: * statistically significant increase of FasL-positive cells among melanotic melanoma cells in comparison with 4-hour-culture; $\mathrm{p}<0.05 .{ }^{* *}$ Statistically significant increase of FasL-positive cells among amelanotic melanoma cells in comparison with 4-hour-culture; statistically significant decrease of FasL-positive cells among amelanotic melanoma cells in comparison with melanotic melanoma cells; $\mathrm{p}<0.05$. ${ }^{* * *}$ Statistically significant decrease of FasL-positive cells among amelanotic melanoma cells in comparison with melanotic melanoma cells; $p<0.05$.

\begin{tabular}{|c|c|c|c|c|}
\hline \multirow{2}{*}{ Transplantable melanoma lines } & \multicolumn{4}{|c|}{ Percentage of Fas- and FasL-positive cells } \\
\cline { 2 - 5 } & & Fas & \multicolumn{3}{|c|}{ FasL } \\
\cline { 2 - 5 } & after 4h & after $24 \mathrm{~h}$ & after $4 \mathrm{~h}$ & after $24 \mathrm{~h}$ \\
\hline Melanotic $(\mathrm{Ma})$ & $3.1 \pm 1.3$ & $2.1 \pm 0.9$ & $11.4 \pm 5.6$ & $32.6 \pm 6.1^{*}$ \\
\hline Amelanotic $(\mathrm{Ab})$ & $4.6 \pm 1.6$ & $3.9 \pm 2.0$ & $1.04 \pm 0.7^{* * * *}$ & $12.9 \pm 5.6^{* * *}$ \\
\hline
\end{tabular}

a posistive control of FasL expression, $51.3 \%$ were FasL-positive. A number of melanotic and amelanotic melanoma cells with FasL expression increased with the extension of incubation time. We observed significant differences in a number of melanoma cells with the FasL expression between melanotic line and more aggressive amelanotic line. About $11.4 \%$ of the native melanotic melanoma cells bound anti-FasL antibody after 4 hours, and about $32.6 \%$ after 24 hours (Table 1; Fig. 2). While among amelanotic melanoma cells only $1.0 \%$ showed FasL expression after 4 hours and this number of cells increased to $12.9 \%$ after 24 hours (Table 1; Fig. 2). Therefore, after 24 hours of incubation FasL appeared on a similar level like initially melanotic melanoma cells had (Table 1).

Observing a dynamics of the FasL expression on cells of both melanoma lines, we could ascertain that after 4 and 24 hours quantitatively number of melanotic melanoma (Ma) cells with the FasL expression is relatively greater in comparison with amelanotic melanoma $(\mathrm{Ab})$ cells. But, in case of amelanotic melanoma $(\mathrm{Ab})$ line, with more aggressive phenotype, the observed increase in the FasL expression was much more dynamic, because we observed 12-fold increase after 24 hours, and in case of melanotic melanoma (Ma) cells, the observed increase of the FasL expression was only about 3 -fold in comparison with the 4-hour-culture.

\section{Discussion}

Melanoma is one of the most aggressive tumours, and mortality caused by melanoma has been on the increase. Molecular mechanisms of this problem are still unknown and are the subject of many studies $[14,15,16]$. One of those mechanisms is the Fas/FasL system, which has been studied for many years $[1,2,25]$. The Fas/FasL system induces cell death pathway via receptors (extrinsic pathway). Many inducers and inhibitors of this way of apoptosis have been reported. It is well recognized that the Fas/FasL pathway is involved in the apoptotic process in different pathologies and in the cytotoxic effects of most immuno- and chemotherapeutic agents [1,2,26,27]. The expression and function of the Fas receptor play an important role in the maintaining of homeostasis. Different types of tumour cells have been shown to escape immune recognition by constitutive resistance to Fas-mediated apoptosis [6,7,25]. Melanoma cells are often characterized by reduced Fas expression and/or death signalling function, rendering them resistant to cell death mediated by FasL $[3,17,27,28,29]$. Due to a higher FasL expression, tumour cells can induce apoptosis of immune cells $[7,17]$. A loss of Fas signaling may occur in melanoma, and FasL upregulation on these tumour cells probably occurs during tumorigenesis [3]. In the case of metastasis, a higher FasL expression on tumor cells could facilitate their migration into tissues surrounding the tumour, throughout the elimination of normal cells with Fas on their surface [7].

The results of the present study indicate that cells of two transplantable melanoma lines expressed Fas receptor at a low level, without any differences between the lines examined, so the Fas expression did not change when the tumour altered from melanotic into amelanotic melanoma line with a more aggressive phenotype. After a longer incubation time, the number of cells with FasL expression changed, and distinct differences were observable between the melanoma lines. The results for cells of both melanoma lines, obtained after 4 hours of incubation, may indicate the contribution of the Fas/FasL system to the induction of spontaneous apoptosis, though only to an insignificant degree (only a few per cent). So, we can say that apoptosis, induced by the Fas/FasL system, does not play a key role in transplantable melanoma cells [20,30], despite the higher ability to undergo spontaneous apoptosis of melanotic melanoma cells in comparison with amelanotic melanoma cells - more aggressive and with a 
$4 \mathrm{~h}$

Ma

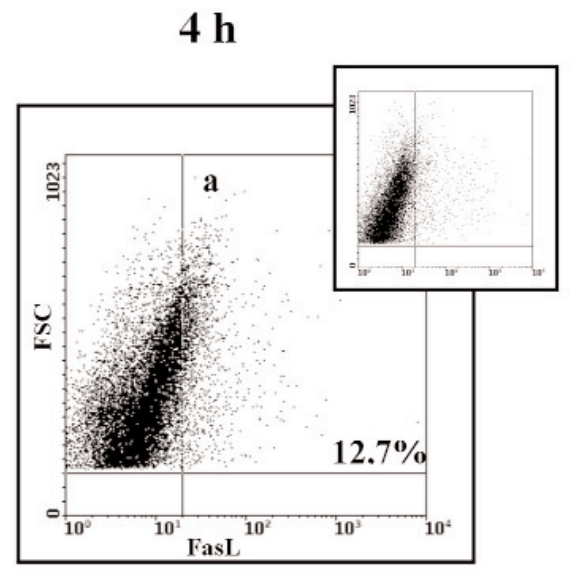

Ab

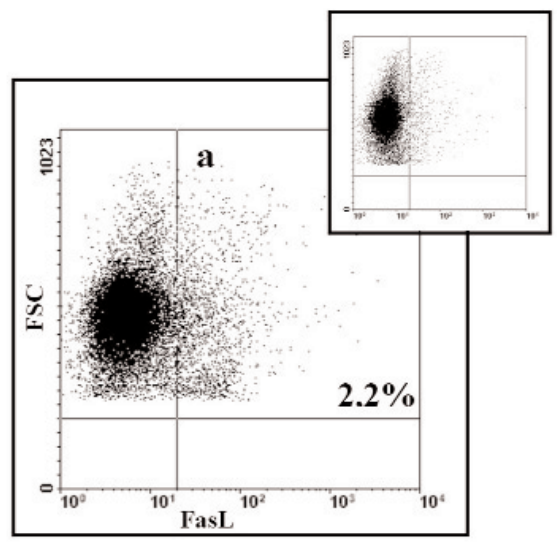

$24 \mathrm{~h}$
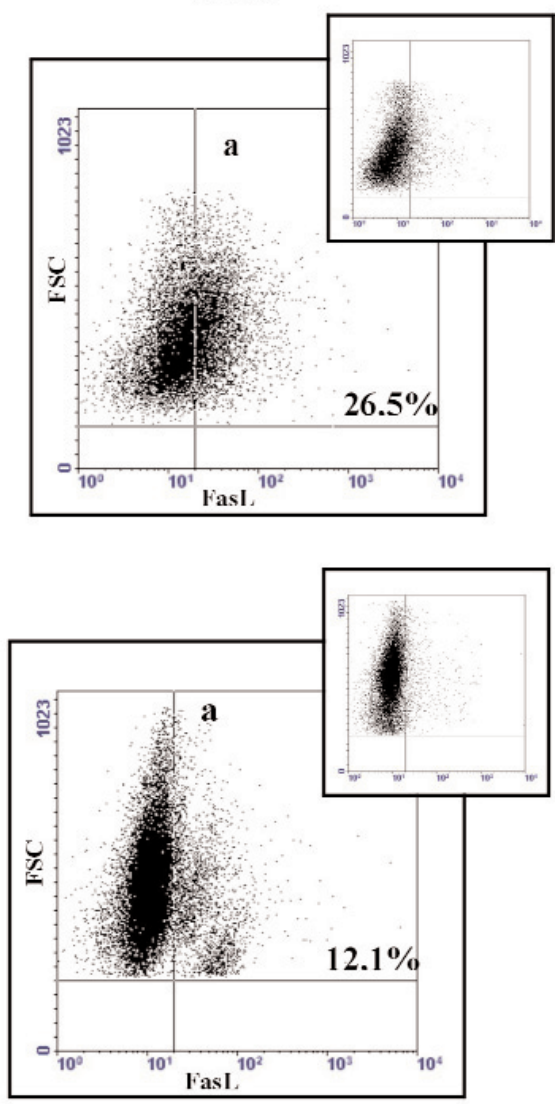

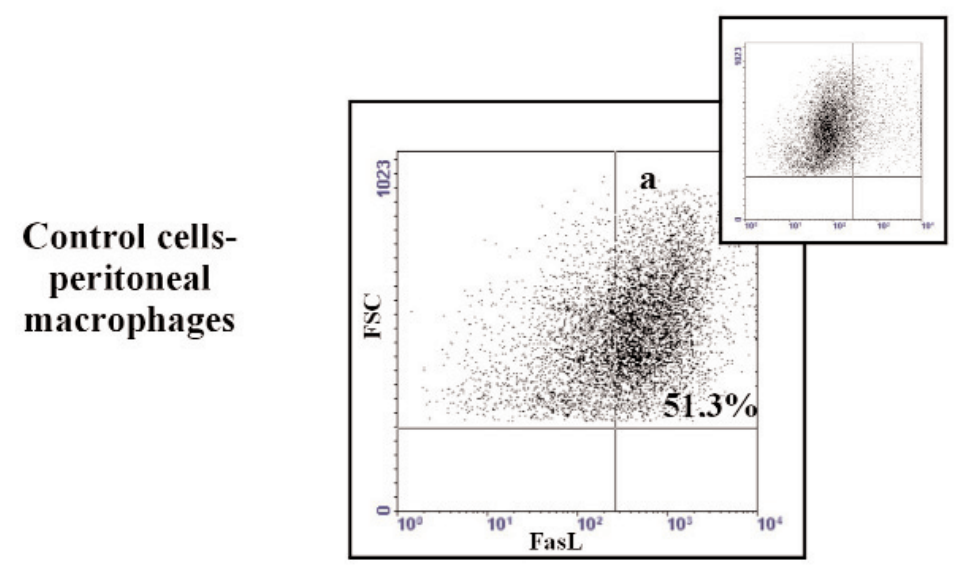

Fig. 2. Expression of FasL on transplantable melanotic $\mathrm{Ma})$ and amelanotic $(\mathrm{Ab})$ melanoma cells. The plots show results of one representative experiment of flow cytometric detection of FasL-positive cells (a - area of FasL-positive melanoma cells). Inserts show fluorescence levels of isotype control. The percentages in the corner of each plot show the positive cells among the population of cells after elimination of background isotype control. Hamster peritoneal macrophages were used as a positive control for the FasL ligand. lower ability to spontaneous apoptosis. Thus, we can suggest that in both transplantable melanoma cell lines the intrinsic pathway of apoptosis plays a more important role than the extrinsic one [18,30].

But, both melanoma lines indicate a considerable difference in the content of FasL-positive cells which was lower in the case of the more aggressive amelanotic line. It might be, as some other authors suggest $[4,31,32]$, caused by the shedding of FasL by amelanotic melanoma cells, a more aggressive and more apoptosis-resistant transplantable melanoma line, to avoid Fas/FasL-induced apoptosis. Wada et al. [33] demonstrated that at high levels FasL triggered an inflammatory response, but at a low level it enhanced tumour growth. The same authors also suggested that FasL expressed in tumours, even at relatively low levels, is capable of killing tumour-infilrating lymphocytes. The above-mentioned observations of Wada A et al. [33], seem to confirm a low FasL-positive cells content in the more aggressive and faster growing amelanotic melanoma line. Thus, a low FasL level may help tumours escape the immune attack. The observed significant differences in the dynamics of FasL expression on cells of both melanoma lines, attendant upon spontaneous alternation of native melanotic melanoma into amelanotic melanoma line with changed biologi- 
cal properties, may suggest that melanoma cells could evade immune control and induce apoptosis of effector immune cells infiltrating the tumour.

Hahne et al. [7], as mentioned earlier, reported that the FasL protein is present in melanoma, and may promote the evasion of antitumour immune responses. However, little or no Fas was expressed by melanoma cells in all patients they examined [7]. In contrast, the majority of cells infiltrating the tumour mass were Faspositive. FasL-expressing melanoma cells (in vitro) induce apoptosis of Fas-sensitive tumour-infiltrating cells at a high rate. Therefore FasL plays an important role in creating the immune privilege of melanoma [7]. No FasL was found in normal melanocytes of the skin, indicating that FasL upregulation probably participates in melanoma tumorigenesis [7]. These results are also convergent with the results of other authors that human and mouse melanoma cells express Fas ligand but hardly any Fas, which may contribute to their immune privilege $[3,34]$. Similar results were presented by Soubrane et al. [28], who determinated the expression of Fas and its ligand (FasL) on normal skin cells (mixture of keratinocytes and melanocytes), and primary and metastatic melanoma cells from patients, using monoclonal antibodies and flow cytometric analysis of labelled cells. They found that Fas expression was significantly higher on normal control skin cells than on cells from tumours, whatever the stage studied. But they observed a significant increase of the FasL expression on melanoma cells compared with normal cells. Also Terheyden et al. [35] observed an increased expression of FasL in cutaneous metastases as compared with their primaries and concluded that FasL expression correlates with the tumorigenicity of melanoma cells, progression of disease and a worse prognosis for the patient.

Our results: a 12-fold increase of FasL-positive amelanotic melanoma cells - with a higher immunogenicity, lower level of cell differentiation, higher growth rate - in comparison with only about 3-fold increase of FasL-positive cells among native melanotic melanoma, seem to suggest the occurrence of immune privilege of amelanotic melanoma cells. The increased dynamics of FasL expression on amelanotic melanoma cells may indicate a possible counterattack against activated immune effector cells such as T cells and NK cells, which could account for the faster growth of amelanotic melanoma cells. The results of other authors, which indicate low Fas and high FasL expression, in human [13] and animal [36] melanomas, jointly confirm our results for two hamster transplantable melanoma cell lines - melanotic and amelanotic.

Tumour expression of FasL has been proposed to aid in immune evasion through a 'Fas counterattack' mechanism, but has also been described as a proin- flammatory factor $[33,37]$. It is not yet fully understood how FasL induces inflammation $[38,39]$. FasL was originally described as a proapoptotic, antiinflammatory protein, but now it is known that FasL can also activate the NFKB pathway and be proinflammatory by mechanisms similar to those for TNF $[40,41]$.

Our previous results $[42,43,44]$ indicate that two melanoma lines differ in the secretion of some cytokines, e.g. TNF, IL-10, which was higher in melanotic melanoma cells. It might be connected with differences in FasL expression level between amelanotic and melanotic melanoma lines.

According to the results of this study and those of other authors, it seems that the Fas/FasL system does not play a key role in inducing spontaneous apoptosis in two transplantable melanoma cell lines. Even if FasL is detected on melanoma cells, its expression does not necessarily predict susceptibility to death. The results presented here support this hypothesis, showing that loss of the Fas expression and/or function and resistance to Fas-mediated apoptosis are frequent both during melanoma development in situ and in melanoma cell lines in vitro. The results obtained could indicate that cells of two transplantable melanoma lines may use Fas/FasL signalling to avoid the immunological attack by increasing the FasL expression and down-regulating the Fas receptor expression.

In conclusion, the findings that indicate changes in the content of FasL-positive cells, attendant upon a spontaneous alteration of transplantable melanoma, show the importance of a complete analysis of FasL expression by tumour cells, in order to fully describe its biological function, and may help resolve the discrepancies present in the literature regarding the Fas/FasL system in melanoma.

\section{References}

[ 1] Walczak H, Krammer PH. The CD95 (APO-1/Fas) and the TRAIL (APO-2L) apoptosis systems. Exp Cell Res. 2000; 256:58-66.

[2] Nagata S. Early work on the function of CD95, an interview with Shige Nagata. Cell Death Differ. 2004;11(suppl.1):2327.

[3] Bullani RR, Wehrli P, Viard-Leveugle I et al. Frequent downregulation of Fas (CD95) expression and function in melanoma. Melanoma Res. 2002;12:263-270.

[ 4] Lee H, Ferguson TA. Biology of FasL. Cytokine Growth Factor Rev. 2003;14:325-335.

[ 5] Uckan D, Steele A, Cherry et al. Trophoblasts express Fas ligand: a proposed mechanism for immune privilege in placenta and maternal invasion. Mol Hum Reprod. 1997;3:655-662.

[ 6] Griffith TS, Brunner T, Fletcher SM et al. Fas ligand-induced apoptosis as a mechanism of immune privilege. Science. 1995;270:1189-1192.

[7] Hahne M, Rimoldi D, Schroter M et al. Melanoma cell expression of Fas (Apo-1/CD95) ligand: implications for tumor immune escape. Science. 1996;274:1363-1366. 
[ 8] Koyama S, Koike N, Adachi S. Fas receptor counterattack against tumor-infiltrating lymphocytes in vivo as a mechanism of immune escape in gastric carcinoma. $J$ Cancer Res Clin Oncol. 2001;127:20-26.

[9] Reimer T, Herrnring C, Koczan D et al. FasL: Fas ratio - a prognostic factor in breast carcinomas. Cancer Res. 2000;60: 822-828.

[10] Niehans GA, Brunner T, Frizelle SP et al. Human lung carcinomas express Fas ligand. Cancer Res. 1997;57:1007-1012.

[11] O'Connell J, O'Sullivan GC, Collins JK et al. The Fas counterattack: Fas mediated T-cell killing by colon cancer cells expressing Fas ligand. J Exp Med. 1996;184:1075-1082.

[12] Debatin KM, Krammer PH. Death receptors in chemotherapy and cancer. Oncogene. 2004;23:2950-2966.

[13] Martinez-Lorenzo MJ, Anel A, Alava MA et al. The human melanoma cell line MelJuSo secretes bioactive FasL and APO2L/TRAIL on the surface of microvesicles. Possible contribution to tumor counterattack. Exp Cell Res. 2004;295 315-329.

[14] Böhm I., Schild H. Apoptosis: the complex scenario for silent cell death. Mol Imaging Biol. 2003;5:2-14.

[15] Hussein MR, Haemel AK, Wood GS. Apoptosis and melanoma: molecular mechanisms. J Pathol. 2003;199:275288.

[16] Kroemer G, El-Deiry WS, Goldstein P et al. Classification of cell death: recommendations of the nomenclature committee on cell death. Cell Death Differ. 2005;12:1463-1476.

[17] Redondo P, Solano T, Vazquez B et al. Fas and Fas ligand: expression and soluble circulating levels in cutaneous malignant melanoma. Br J Dermatol 2002;147:80-86.

[18] Cichorek M, Kozłowska K, Wachulska M et al. Spontaneous apoptosis of melanotic and amelanotic melanoma cells in different phases of cell cycle: relation to tumor growth. Folia Histochem Cytobiol. 2006;44:31-36.

[19] Kozłowska K, Cichorek M, Zarzeczna M et al. Heterogeneous susceptibility to spontaneous and induced apoptosis characterizes two related transplantable melanomas with different biological properties. Pigment Cell Res. 2002;15:233238.

[20] Zielińska K, Kozłowska K, Cichorek M et al. Participation of the Fas/FasL system in spontaneous apoptosis of two transplantable melanoma line cells. Folia Histochem Cytobiol. 2005;43(suppl.1):75-76. Abstracts from IX Conference on Cell Biology, 15-17 September, 2005, Łódź, Poland.

[21] Bomirski A, Słomiński A, Bigda J. The natural history of a family of transplantable melanomas in hamsters. Cancer Metastasis Rev. 1988;7:95-118.

[22] Bomirski A. A biological properties of transplantable melanomas in the Syrian hamster during 16 years of maintenance by serial passages. Acad Med Ged. 1977.

[23] Bomirski A, Dominiczak T, Nowińska L. Spontaneous transplantable melanoma on golden hamster. Acta Union Int Cancer. 1962;18:178-180.

[24] Bomirski A, Kozłowska K, Żurawska-Czupa B. An attempt to prepare nonenzymatically, a single cell suspension from solid transplantable melanomas. Proceedings of the IXth International Conference of Pigment Cell. 1975;5-6, Texas, Houston.

[25] Raisova M, Bektas M, Wieder T et al. Resistance to CD95/Fas-induced and ceramide-mediated apoptosis of human melanoma cells is caused by a defective mitochondrial cytochrome c release. FEBS Lett. 2000;473:27-32.

[26] Gutierrez LS, Noria F, Finol H et al. Fas ligand expression and its correlation with apoptosis and proliferation in Lobund-Wistar prostate carcinomas. Pathobiology. 2005;72: 260-268.
[27] Shukuwa T, Katayama I, Koji T. Fas-mediated apoptosis of melanoma cells and infiltrating lymphocytes in human malignant melanomas. Mod Pathol. 2002;15:387-396.

[28] Soubrane C, Mouawad R, Antoine EC et al. A comparative study of Fas and Fas-ligand expression during melanoma progression. Br J Dermatol. 2000;143:307-312.

[29] Tada Y, O-Wang J, Wada A et al. Fas ligand-expressing tumors induce tumor-specific protective immunity in the inoculated hosts but vaccination with the apoptotic tumors suppresses antitumor immunity. Cancer Gene Ther. 2003;10: 134-140.

[30] Cichorek M, Kozłowska K, Bryl E. Mitochondrial transmembrane potential in spontaneous and camptothecin-induced apoptosis of melanotic and amelanotic melanoma cells. Neoplasma. 2007;54:29-36.

[31] Hohlbaum AM, Moe S, Marshak-Rothstein A. Opposing effects of transmembrane and soluble Fas ligand expression on inflammation and tumor cell survival. $J$ Exp Med. 2000; 191:1209-1219.

[32] Tanaka M, Itai T, Adachi M et al. Downregulation of Fas ligand by shedding. Nat Med. 1998;4:31-36.

[33] Wada A, Tada Y, Kawamura K et al. The effects of FasL on inflammation and tumor survival are dependent on its expression levels. Cancer Gene Ther. 2007;14:262-267.

[34] Kalechman Y, Strassmann G, Albeck M et al. Up-regulation by ammonium trichloro(dioxoethylene- $0,0^{\prime}$ ) tellurate (AS101) of Fas/Apo-1 expression on B16 melanoma cells: implications for the antitumor effects of AS101. J Immunol. 1998; 161:3536-3542.

[35] Terheyden P, Siedel C, Merkel A et al. Predominant expression of Fas (CD95) ligand in metastatic melanoma revealed by longitudinal analysis. J Invest Dermatol. 1999;112:899-902.

[36] Peter I, Mezzacasa A, LeDonne P et al. Comparative analysis of immunocritical melanoma markers in the mouse melanoma cell lines B16, K1735, S91-M3. Melanoma Res. 2001;11:2130.

[37] Chen JJ, Sun Y, Nabel GJ. Regulation of the proinflammatory effects of Fas ligand (CD95L). Science. 1998;282:1714-1717.

[38] Restifo NP. Not so Fas: re-evaluating the mechanisms of immune privilege and tumor escape. Nat Med. 2000;6:493-495.

[39] Ryan AE, Lane S, Shanahan F et al. Fas ligand expression in human and mouse cancer cell lines; a caveat on over-reliance on mRNA data. J Carcinog. 2006;5:5.

[40] Aggarwal BB, Shishodia S, Sandur SK et al. Inflammation and cancer: how hot is the link?. Biochem Pharmacol. 2006; 72:1605-1621.

[41] Philip M, Rowley DA, Schreiber H. Inflammation as a tumor promoter in cancer induction. Semin Cancer Biol. 2004; 14:433-439.

[42] Kozłowska K, Zarzeczna M, Cichorek M. Characterization of NO and cytokine release by transplantable melanoma cell lines in relationship to their differentiation. Arch Immunol Ther Exp. 2001;49(supp1.2):103-109.

[43] Kozłowska K, Zarzeczna M, Cichorek M et al. Characterization of interleukin 10 and NO release by transplantable melanoma cell lines with regard to their progression. NeOplasma. 2003;50:85-90.

[44] Wachulska M, Kozłowska K, Cichorek M. The DNA ploidy and proliferative activity of transplantable melanoma cells in regard to their secretory function. Neoplasma. 2005;52:280286.

Submitted: 20 December, 2007 Accepted after reviews: 27 April, 2008 